\title{
Visual Search for Emotional Facial Expression: Effect of Space Density on Visual Crowd Effect
}

\author{
Siqi Huang ${ }^{1, a, \uparrow, *}$, Xinqian $\mathrm{Li}^{2}, \mathrm{~b}, \uparrow, *$, Jiaqin $\mathrm{Lu}^{3, \mathrm{c}, \uparrow, *}$ \\ ${ }^{1}$ Beijing Sport University, Beijing, China \\ ${ }^{2}$ Chongqing Normal University, Chongqing, China \\ ${ }^{3}$ Nanjing Normal University, Nanjing, Jiangsu, China \\ *Corresponding author. Email: ${ }^{a} 1810072 @$ bsu.edu.cn, ${ }^{b} 2018051603090 @$ stu.cqnu.edu.cn, ${ }^{c} 01180104 @$ njnu.edu.cn \\ ${ }^{\dagger}$ These authors contributed equally.
}

\begin{abstract}
Visual search studies have found that positive facial expressions are more efficiently detected among a crowd of distractor faces than other expressions, known as the happy superiority effect (HSE). Moreover, the density of peripheral items in clutter can also impact our process of visual search. The present study investigated the HSE among different space densities in a visual crowding task. In the experiment, the stimuli consist of 12 facial images, and a 2 (target emotion: positive vs. negative) $\times 3$ (space density: null vs. low vs. high) $\times 3$ (flanker emotion: neutral vs. positive vs. negative) within subject design was conducted. Participants were instructed to determine whether the target face in the centre was positive or negative. Results showed that space density had a significant effect. Generally, the greater the space density was, the slower the recognition speed was, reflecting that crowding conditions needed more attentional resources, leading to more mistakes in recognition processing. And the HSE in light crowding conditions was the most robust, but not in heavy conditions. There was a gradual change from a weak HSE to a strong HSE as the space density became smaller. In conclusion, our findings contributed to the study of visual search and emotion recognition, creatively making introduction of space density, which filled the emptiness of previous studies and reducing the limitation of schematic faces in real-life situation, but still needed further investigation.
\end{abstract}

Keywords: Emotional facial expression, Space density, Visual crowd effect, Visual search

\section{INTRODUCTION}

Human faces display many signals that guide interpersonal communication, such as expressions of emotion and intention, making them incredibly important sources of social information. Happy smiles provide invitations for engaging in cooperation and interaction, whereas angry faces can be perceived as warning signals of a possible threat. Identifying emotional expressions is one of the most prominent mechanisms helping individuals judge other people's intentions and predict their behaviours [1]. Furthermore, in many situations, people do not perceive only a single face but faces in the crowd. It is more typical for a daily life situation that crowds with more target emotions, for example, when giving a speech in front of lots of people [2].

\subsection{Emotion superiority effect}

Previous research on the preferential processing of emotional facial expressions in visual search has obtained different results [3]. A number of researchers have reported the anger superiority effect (ASE), which means angry target faces were found faster than happy target faces and appeared to "pop-out" of crowds with distractor expressions [4]. This pattern is interpreted as evidence for prior detection of threat-related cues in the environment [5].

However, there is also evidence to find the opposite outcome, the happiness superiority effect (HSE) [2, 3, 6]. One explanation is that human expressions of happiness have a more obvious communicative intent, and are therefore more visually discriminable [3]. A metaanalysis study [7] comparing ASE and HSE found the recognition advantage for happy faces. Especially in emotion recognition tasks, an advantage in recognition accuracy and speed for happy expressions was found for all stimulus types. 


\subsection{Visual crowd effect}

Emotional facial search is just part of the visual search process in the real context. It is reported that peripheral items in clutter have an impact on our process of visual search. The crowding effect has been found with various stimuli, including faces $[8,9]$. Crowding disrupts object recognition, but interestingly facial expressions can break through the suppression of crowding largely [10]. In reality, a growing body of evidence suggests that facial expressions survive crowding even when they are not consciously accessible [11]. Therefore, based on the works of early researchers, we assume that visual crowding may influence emotional face recognition.

The crowding effect, the inability to identify objects in clutter, renders a fundamental limit on conscious visual perception and object recognition throughout most visual fields [12]. This effect is proposed based on Bouma law. Our ability to recognize an object depends on the ratio of the spacing between the object and the similar surrounding objects to the observer's critical spacing at that location [13]. Many variables influence the phenomenon, such as the position of the stimuli (innerouter asymmetry) [14], the appearance time of the stimuli (Temporal tuning) [15]. There is evidence that space density plays a crucial role in the visual search effect. At a specific eccentricity, identification of a crowded target improves as the distance between the target and flankers increases (lower space density) [12, 13].

However, most of the finding of the visual crowding effect used non-social materials like gabor and houses. Too little work has been devoted to social materials. Only a limited number of studies used real faces in the visual crowd task. Bucher and Voss [2] used different number of emotional faces to manipulate the level of crowding and find the happiness superiority effect. Sun and Balas [16] find that the different crowding degrees of both Chinese characters and electrical socket as flanker will affect the recognition of the real face emotion. Moreover, crowding faces also affected emotional judgments of neutral stimuli (Chinese characters). Farzin et al. [8] used the Mooney faces as material and flanker spacing as a variable in their study. The result showed recognition of the orientation of the face decreased significantly as spacing decreased. Recently, one study [17] combined the anger superiority effect and visual crowd effect and found ASE only when flankers were upright neutral faces. Nevertheless, there is no solid conclusion on how the emotional superiority effect and visual crowd effect interact.

\subsection{Current study}

There is no shortage of ideas about the explanation for space density on crowding from low-level receptive field models [18-22], but too little work has been devoted to social recognition. To our knowledge, no study to date has examined the space density on the emotional visual search task. Moreover, emotional face preference is an inevitable effect of emotional face recognition. The emotion superiority effect has received considerable critical attention, but still, no consistent conclusion was found.

Therefore, for our first goal, we want to investigate the effect of anger and happiness priority. First, we hypothesize that there is a superiority for positive face. This research sheds new light on whether the space density of the surrounding distracted faces will influence the visual crowd effect and how the emotional priority effect interacts with this process. We hypothesize that the effect of crowding should be less when space density is lower, which means the shorter time and higher accuracy of response.

\section{METHOD}

\subsection{Participants}

40 Chinese college students from Beijing Sport University, Nanjing Normal University, and Chongqing Normal University participated in our experiment, including 16 males and 24 females. The mean age of the sample was 20.85 years $(S D=0.69)$. The subjects had normal vision or corrected vision, no colour blindness, right-handed, and had never done similar experiments before.

\subsection{Stimuli}

The experimental stimuli consisted of 12 images of human faces, obtained from the Chinese Affective Picture System Database (CAPS, [23]). Three types of facial expressions-neutral, positive, and negative Chinese faces (4 pictures each) were selected for this experiment. Each image was cropped so that it included only the head and was standardized in size.

\subsection{Design and procedure}

The experiment involves two conditions: low congestion and high congestion. When the centre of the screen is represented by the coordinate $(0,0)$, the Flanker and the target are separated by 0.1 units under the condition of high crowding. Under low crowding conditions, the interval between the two was 0.3 units. Two target emotions (positive and negative) and three flanking emotions (neutral, positive, and negative) were used for each trial. Each experiment consists of two parts: the test part (36 trials) and the formal experiment (144 trials). The whole experiment takes about 10 minutes and is implemented by Psychopy3. The flow chart is as follows (see Figure 1). 


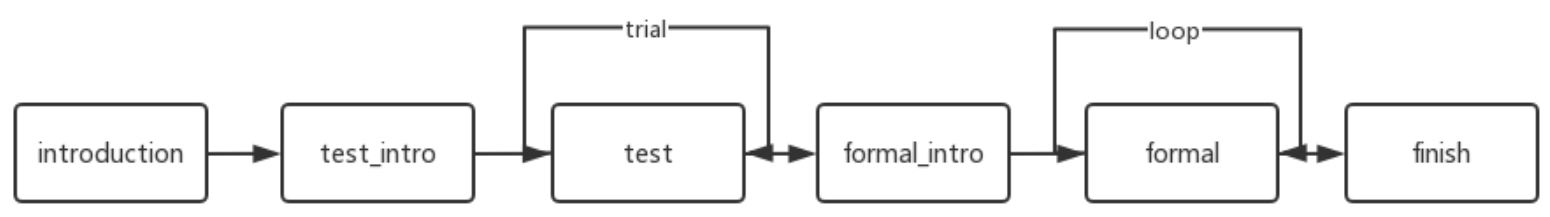

Figure1. Experimental flow chart.

This experiment has three independent variables: space density (null, low, high), flanker emotion (positive, neutral, negative), target emotion (positive, negative). The extraneous variables are location, the gender of the material. Meanwhile, we controlled the independent variables uniformly (such as the frequency of occurrence of various positions, the frequency of conditional repetition, the size of targets and flanks, and the gender repetition of materials) to eliminate the impact on the accuracy of the experiment.

\subsection{Trail}

On each trial of the crowded condition, a fixation point was displayed in the centre of the screen for 1000 $\mathrm{ms}$, followed by the face stimuli that appeared either on the left or right side of the screen for $400 \mathrm{~ms}$ (see Figure 2 ). The brief presentation time and random location of the target could avoid any eye movement toward the target.

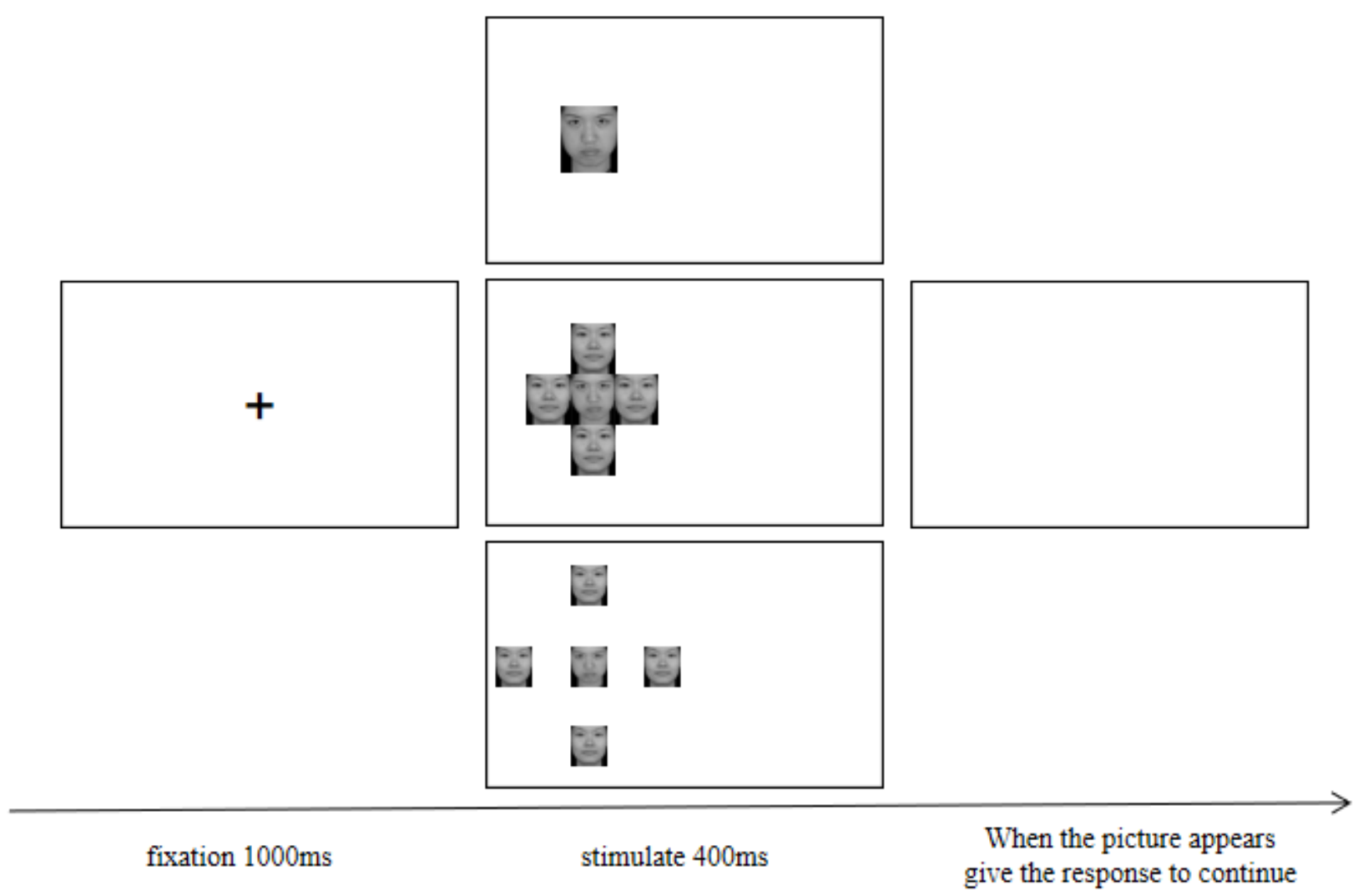

Figure2. Schematic illustration of a trial in the experiment.

Participants were presented with a screen with a white cross in the middle, and then photographs of several faces were shown to the left or right, and they were asked to rate the emotions of the central face and ignore the others. When a positive face was presented, press the F key. When a negative face was presented, press the $\mathrm{J}$ key. In addition, the participants were asked to respond as quickly and accurately as possible.

\subsection{Statistical analysis}

In this experiment, SPSS 25.0 was used to analyze the data, based on a three-factor repeated measures ANOVA. 
Data from participants who made more errors than 3 standard deviations from the mean were discarded.

\section{RESULTS}

\subsection{Reaction Time}

The data analysis was based on reaction times for correct responses. The mean RT of different conditions is displayed in Table 1.

Table 1. The mean RT (ms) in different conditions.

\begin{tabular}{llll}
\hline Target & Space & Flanker & M \pm SD \\
Emotion & Density & Emotion & \\
\hline Negative & null & Neutral & $757.18 \pm 165.70$ \\
& & Positive & $776.86 \pm 163.06$ \\
& & Negative & $765.30 \pm 140.83$ \\
& low & Neutral & $823.48 \pm 153.15$ \\
& & Positive & $820.54 \pm 193.88$ \\
& & Negative & $805.96 \pm 143.63$ \\
& high & Neutral & $812.77 \pm 168.29$ \\
& & Positive & $815.54 \pm 161.60$ \\
Positive & null & Negative & $806.63 \pm 193.06$ \\
& & Neutral & $751.71 \pm 135.17$ \\
& & Positive & $736.19 \pm 140.85$ \\
& low & Negative & $731.04 \pm 133.37$ \\
& & Neutral & $784.34 \pm 142.95$ \\
& & Positive & $774.31 \pm 146.61$ \\
& high & Negative & $781.51 \pm 129.50$ \\
& & Neutral & $804.43 \pm 160.44$ \\
& & Positive & $795.43 \pm 161.07$ \\
& & Negative & $825.16 \pm 178.77$ \\
\hline
\end{tabular}

A 2 (target emotion: positive vs. negative) $\times 3$ (space density: null vs. low vs. high) $\times 3$ (flanker emotion: neutral vs. positive vs. negative) repeated measures ANOVA was conducted on mean RT in different conditions. Results showed that the main effect of target emotion approached significance $(\mathrm{F}(1,39)=3.425, \mathrm{p}=$ $\left.0.072, \eta_{\mathrm{p}}{ }^{2}=0.081\right)$, reflecting the fact that the mean reaction time of positive target faces $(776 \mathrm{~ms} \pm 20$ $\mathrm{ms})$ was faster than negative target faces $(798 \mathrm{~ms} \pm 24$ $\mathrm{ms})$.Also, the effect of space density $(\mathrm{F}(2,78)=29.314$, $\left.\mathrm{p}<0.001, \eta_{\mathrm{p}}{ }^{2}=0.429\right)$ was significant, which showed that reaction in the uncrowded position $(753 \mathrm{~ms} \pm 20 \mathrm{~ms})$ was the quickest, followed by slightly crowded (798 ms $\pm 21 \mathrm{~ms})$ and heavily crowded $(810 \mathrm{~ms} \pm 24 \mathrm{~ms})$. However, there was no significant difference between positive, negative, or neutral flankers $(F(2,62)=0.095$, $\mathrm{p}=0.865)$.

In addition, the three-way interaction was not significant $(F(3,116)=0.78, p=0.507)$, but the two-way interaction between target emotion and space density was significant marginally $\left(\mathrm{F}(2,78)=2.796, \mathrm{p}=0.067, \eta_{\mathrm{p}}{ }^{2}=\right.$ $0.067)$. When the targets were uncrowded, the mean RT of positive faces was significantly lower than negative faces $(p=0.070)$, also in the light condition $(p=0.013)$, see Figure 3. No significant differences were found between the facial expressions of the two targets when heavy crowded $(\mathrm{p}=0.831)$.

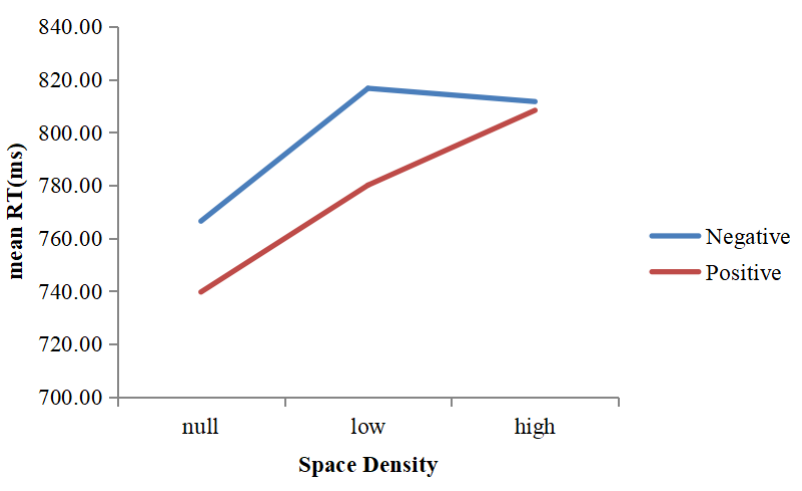

Figure 3. Results of the RT analysis. The two-way interaction between target emotion and space density.

\subsection{Accuracy}

Percentages of correct trials averaged across subjects were computed when there were no flankers (uncrowded condition) and flankers (two crowded conditions). Results are plotted in Figure 4.

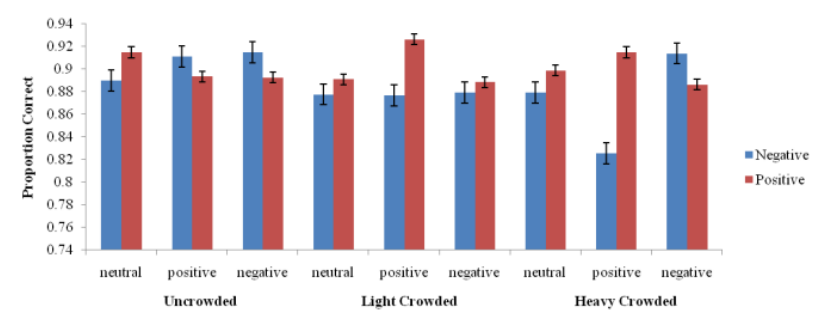

Figure 4. Result of the accuracy analysis. The y-axis indicates the proportion of correct facial expressions recognition. Error bars indicate \pm 1 SEM.

A 2 (target emotion: positive vs. negative) $\times 3$ (space density: null vs. low vs. high) $\times 3$ (flanker emotion: neutral vs. positive vs. negative) repeated measures ANOVA was conducted on mean accuracy in different conditions. Results showed that the effect of space density approached significance $(\mathrm{F}(2,78)=3.022, \mathrm{p}=$ $\left.0.054, \eta_{\mathrm{p}}{ }^{2}=0.072\right)$, reflecting the fact that the percentage of correct recognition in the uncrowded position (91.5\%) was the highest, followed by slightly crowded $(90.5 \%)$ and heavily crowded $(89.6 \%)$. However, the effect of target emotion $(\mathrm{F}(1,39)=0.026, \mathrm{p}=0.872)$ and flanker emotion $(\mathrm{F}(2,78)=0.037, \mathrm{p}=0.964)$ were both not significant.

In addition, the three-way interaction was not significant $(\mathrm{F}(4,156)=1.354, \mathrm{p}=0.252)$. Still, the twoway interaction between target emotion and flanker emotion was significant marginally $(\mathrm{F}(2,78)=3.001$, p $\left.=0.055, \eta_{\mathrm{p}}{ }^{2}=0.071\right)$. But no significant differences were found between different facial expressions of flankers when the targets were uncrowded, light crowded or heavy crowded, see Figure 5. 


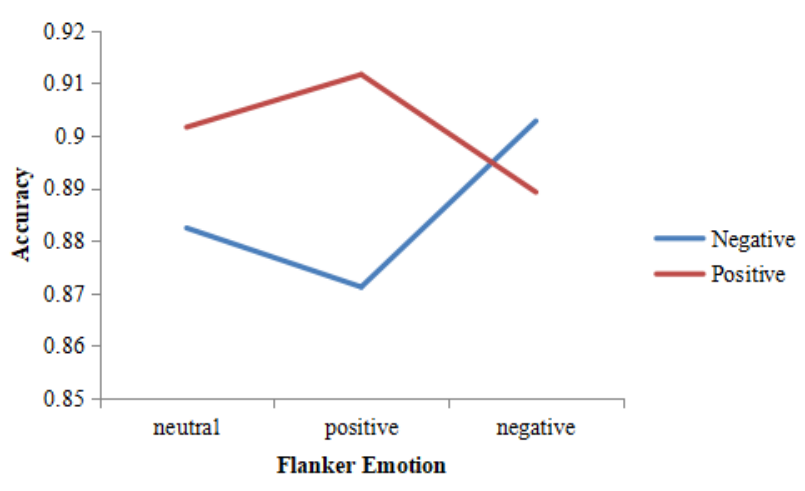

Figure 5. Result of the accuracy analysis. The two-way interaction between target emotion and flanker emotion.

\section{DISCUSSION}

\subsection{Findings}

Results showed that positive faces aroused more attention than negative faces, reflected in both reaction time and accuracy. On the one hand, the reaction time of positive faces was less than that of negative faces, suggesting that positive faces were easier found in the crowded and better break through the suppression of crowding than negative faces. In other words, positive information had priority over emotional information. There was a possible explanation that according to Becker [3], happy expression detectors constantly scan attended objects for positive human affordances, which cause human expression of happiness being evolved to be more visually discriminable. On the other hand, the accuracy of positive faces was higher than that of negative faces, proving that people could recognize faces with positive emotions more accurately, which exactly means the superiority of positive faces in face recognition.

Our results confirmed the hypothesis that positive faces (HSE) have a superiority effect, which replicated many earlier findings [3, 7]. Although our results were not perfect, they could show the tendency of the effect. Results showed that the speed of recognition was quickest when the targets were uncrowded, followed by light crowded and heavy crowded conditions. As a result, the space density of flankers was significantly affecting face recognition, proving the existence of the Visual Crowding Effect. In addition, the result of accuracy analysis was the same. The accuracy of recognition was quickest when the targets were uncrowded, followed by light crowded and heavy crowded condition, reflecting that faces without crowding were more advantaged to accurate recognition. The higher space density was, the less accurate face recognition. The strength model of selfcontrol [24-26] could possibly explain the result that crowding conditions needed more attentional resources, leading to more mistakes in recognition processing.
Whether and what kind of emotional information the flanker had were suggested that would affect the face recognition, but no strong evidence was found in results. Previous studies had argued that there were several extra influencing variables, such as the emotional valence of flankers $[8,9,16]$ and so on.

The HSE was not significant in all crowding conditions. It could be found that the HSE was most evident in light crowding conditions but not in heavy crowding conditions, due to the increased number of flankers which could enhance the interference. The possible reason was that fewer flankers somehow highlighted the target stimulus, making HSE more obvious. Thus, the number of flankers might be a moderating variable of the experiment.

As for the crowding effect, when the target was positive, the greater the space density was, the slower the recognition speed was, but when the target was negative, the speed in light crowding conditions was the slowest. Possibly, ASE and HSE were not exclusive but exhibited in different conditions, according to the Attentional Demands Modulation hypothesis proposed by Gong and Smart [17]. This hypothesis indicates that different tasks demand implies different cognitive loads. Participants will not have sufficient cognitive resources for the task when the cognitive load is overburdened, which can bias their cognitive processing [27-28]. Specifically, there tends to be an ASE when a task requires high attentional demand (e.g., when the target is crowded). While there is no difference in recognising angry and happy faces when attentional demand is medium (e.g., uncrowded condition in the current study) and an HSE when the attentional demand is low [29].

Some factors possibly affect the results. First, we chose CAPS (Chinese Affective Picture System, [23]) as our experiment material. Still, due to limited time and energy, we only used 12 pictures from 4 people ( 2 male and 2 female), and that could bring unexpected extra variables.

Second, the emotional valence of the target picture had not been tested, which could influence the experiment effect and participants' emotional state [30]. For example, one of the materials had led to confusion, according to some participants, they were not sure about the expression of this face, let alone which key to press. Another possible reason was that participants tended to feature search, which means just searching for a single feature. For example, the confusion came out when there was a smiling mouth and crying eyes in one picture.

Third, the low-level perceptual features of real human faces could influence the effect. According to Nummenmaa and Calvo [7], there was an ASE for schematic faces, but a HSE was more likely to show for photographic faces. For example, the mouth feature of faces could cause a misunderstanding that "teeth mean 
happy", so participants just searched for teeth and visualized teeth as happy faces [30-31]. Also, different levels of facial attractiveness could disturb recognition process [32]. Moreover, using random combinations of distractor faces that were neither happy nor angry, researchers could create a heterogeneous background to control non-emotion relevant differences in low-level perceptual features [33]. The heterogeneous background was created by combining the eyebrow and mouth features of the happy and angry targets. One type of distractor face had happy eyebrows but an angry mouth (sad), and the other had angry eyebrows but a happy mouth (scheming). Thus, some researchers have chosen schematic faces, which are simple lines drawing emotional faces, as materials to eliminate the confounding influence of real human faces, but schematic faces have been consistently criticized for lacking ecological validity [6] that could not generalized to real-life situations. As a result, our experiment chose real human faces as experimental materials, trying to imitate the recognition process of faces in reality, and to generalize observed behaviour in the laboratory to natural behaviour of everyone.

\subsection{Limitations}

There were several limitations in our experimental design. On the one hand, no necessary break could cause participants fatigue and influence their emotional state, concentration level, etc. On the other hand, our materials repeatedly appeared, and participants could recognize the person in the picture instead of the expression. In other words, the familiarity of the target picture could bring interference [34]. Furthermore, the experiment lacked necessary control of individual differences in participants and extra variables, such as search strategies (feature search, just searching for a single feature or configural/holistic processing, [35]) and so on.

In addition, it was a small sample size in our study that only included 40 participants, which may not be enough for more meaningful results. Additionally, lacking further analysis also reduces the validity of our results. RT of wrong responses was all directly deleted in the data analysis, resulting in the data of two participants were discarded as unreliable, which could affect the homogeneity of data.

We have not come to relatively effective or innovative results for limitations in materials, experimental design, and statistical analysis, which calls for further essential exploration.

\subsection{Future directions}

The innovation of our study was the introduction of space density, which reduced the emptiness of previous studies. Further study might consider adding a few more independent variables or change several current variables based on the current research, such as specific search strategy, the availability of attentional resources, and so on.

\section{CONCLUSION}

In conclusion, generally, the greater the space density was, the slower the recognition speed was, reflecting that crowding conditions needed more attentional resources, leading to more mistakes in recognition processing. And the HSE in light crowding conditions was the most robust, but not in heavy conditions. Possibly there was a gradual change from a weak HSE to a strong HSE as the space density became smaller. These findings were good news for not only social psychology researchers but developers of facial recognition software.

\section{REFERENCES}

[1] Calvo, M. G., \& Marrero, H. 2009. Visual search of emotional faces: The role of affective content and featural distinctiveness. Cognition and Emotion, 23(4), 782-806.

[2] Bucher, Alica, and Andreas Voss. 2019. 'Judging the Mood of the Crowd: Attention Is Focused on Happy Faces.' Emotion 19 (6): 1044-59. https://doi.org/10.1037/emo0000507.

[3] Becker, D. Vaughn, Uriah S. Anderson, Chad R. Mortensen, Samantha L. Neufeld, and Rebecca Neel. 2011. 'The Face in the Crowd Effect Unconfounded: Happy Faces, Not Angry Faces, Are More Efficiently Detected in Single- and Multiple-Target Visual Search Tasks.' Journal of Experimental Psychology: General 140 (4): 63759. https://doi.org/10.1037/a0024060.

[4] Hansen, Christine H., and Ranald D. Hansen. 1988. 'Finding the Face in the Crowd: An Anger Superiority Effect.' Journal of Personality and Social Psychology 54 (6): 917-24. https://doi.org/10.1037/0022-3514.54.6.917.

[5] Fox, Elaine, and Ljubica Damjanovic. 2006. 'The Eyes Are Sufficient to Produce a Threat Superiority Effect.' Emotion 6 (3): 534-39. https://doi.org/10.1037/1528-3542.6.3.534.

[6] Juth, Pernilla, Daniel Lundqvist, Andreas Karlsson, and Arne Öhman. 2005. 'Looking for Foes and Friends: Perceptual and Emotional Factors When Finding a Face in the Crowd.' Emotion 5 (4): 379 95. https://doi.org/10.1037/1528-3542.5.4.379.

[7] Nummenmaa, Lauri, and Manuel G. Calvo. 2015. 'Dissociation between Recognition and Detection Advantage for Facial Expressions: A MetaAnalysis.' Emotion $15 \quad$ (2): 243-56. https://doi.org/10.1037/emo0000042. 
[8] Farzin, F., S. M. Rivera, and D. Whitney. 2009. 'Holistic Crowding of Mooney Faces'. Journal of Vision 9 (6): 18-18. https://doi.org/10.1167/9.6.18.

[9] Louie, Elizabeth G., David W. Bressler, and David Whitney. 2007. 'Holistic Crowding: Selective Interference between Configural Representations of Faces in Crowded Scenes'. Journal of Vision 7 (2): 24. https://doi.org/10.1167/7.2.24.

[10] Kouider, Sid, Vincent Berthet, and Nathan Faivre. 2011. 'Preference Is Biased by Crowded Facial Expressions'. Psychological Science 22 (2): 18489. https://doi.org/10.1177/0956797610396226.

[11] Manassi, Mauro, and David Whitney. 2018. 'MultiLevel Crowding and the Paradox of Object Recognition in Clutter'. Current Biology 28 (3): R127-33. https://doi.org/10.1016/j.cub.2017.12.051.

[12] Whitney, David, and Dennis M. Levi. 2011. 'Visual Crowding: A Fundamental Limit on Conscious Perception and Object Recognition'. Trends in Cognitive Sciences 15 (4): 160-68. https://doi.org/10.1016/j.tics.2011.02.005.

[13] Bouma, H. 1970. 'Interaction Effects in Parafoveal Letter Recognition'. Nature 226 (5241): 177-78. https://doi.org/10.1038/226177a0.

[14] Shechter, Adi, and Amit Yashar. 2021. 'Mixture Model Investigation of the Inner-Outer Asymmetry in Visual Crowding Reveals a Heavier Weight towards the Visual Periphery'. Scientific Reports 11 (1): 2116. https://doi.org/10.1038/s41598-02181533-9.

[15] Chakravarthi, Ramakrishna, and Patrick Cavanagh. 2007. 'Temporal Properties of the Polarity Advantage Effect in Crowding'. Journal of Vision 7 (2): 11. https://doi.org/10.1167/7.2.11.

[16] Sun, Hsin-Mei, and Benjamin Balas. 2015. 'Face Features and Face Configurations Both Contribute to Visual Crowding'. Attention, Perception, \& $\begin{array}{llll}\text { Psychophysics } & 77 & \text { (2): } & 508-19 .\end{array}$ https://doi.org/10.3758/s13414-014-0786-0.

[17] Gong, Mingliang, and L. James Smart. 2021. 'The Anger Superiority Effect Revisited: A Visual Crowding Task'. Cognition and Emotion 35 (2): 214-24. https://doi.org/10.1080/02699931.2020.1818552.

[18] Chung, S. T., D. M. Levi, and G. E. Legge. 2001. 'Spatial-Frequency and Contrast Properties of Crowding'. Vision Research 41 (14): 1833-50. https://doi.org/10.1016/s0042-6989(01)00071-2.
[19] Intriligator, J., and P. Cavanagh. 2001. 'The Spatial Resolution of Visual Attention'. Cognitive Psychology $43 \quad$ (3): $171-216$. https://doi.org/10.1006/cogp.2001.0755.

[20] Levi, Dennis M. 2008. 'Crowding-An Essential Bottleneck for Object Recognition: A MiniReview'. Vision Research 48 (5): 635-54. https://doi.org/10.1016/j.visres.2007.12.009.

[21] Pelli, D. G., Melanie Palomares, and Najib J. Majaj. 2004. 'Crowding Is Unlike Ordinary Masking: Distinguishing Feature Integration from Detection'.

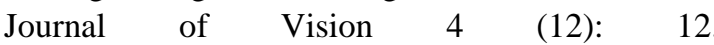
https://doi.org/10.1167/4.12.12.

[22] Toet, A., and D. M. Levi. 1992. 'The TwoDimensional Shape of Spatial Interaction Zones in the Parafovea'. Vision Research 32 (7): 1349-57. https://doi.org/10.1016/0042-6989(92)90227-a.

[23] Bai, L., Ma, H., Huang, Y., \& Luo, Y. 2005. The Development of Native Chinese Affective Picture System--A pretest in 46 College Students. Chinese Mental Health Journal, 19(11), 719-722.

[24] Baumeister, R. F., Bratslavsky, E., Muraven, M., \& Tice, D. M. (1998). Ego depletion: Is the active self a limited resource? Journal of personality and social psychology, 74(5), 1252.

[25] Baumeister, R. F. (2002). Yielding to temptation: Self-control failure, impulsive purchasing, and consumer behavior. Journal of consumer Research, 28(4), 670-676.

[26] Baumeister, R. F. (2002). Ego depletion and selfcontrol failure: An energy model of the self's executive function. Self and identity, 1(2), 129-136.

[27] Casa de Calvo, M. P., \& Reich, D. A. (2007). Spontaneous correction in the behavioral confirmation process: The role of naturallyoccurring variations in self-regulatory resources. Basic and applied social psychology, 29(4), 351364.

[28] Casa de Calvo, M. P., \& Reich, D. A. (2009). Detecting perceiver expectancies: The role of perceiver distraction in spontaneously triggering identity negotiation. Basic and applied social psychology, 31(2), 174-187.

[29] Elfenbein, H. A., \&Ambady, N. (2002). On the universality and cultural specificity of emotion recognition: a meta-analysis. Psychological bulletin, 128(2), 203.

[30] Horstmann, G., Lipp, O. V., \& Becker, S. I. (2012). Of toothy grins and angry snarls-Open mouth 
displays contribute to efficiency gains in search for emotional faces. Journal of Vision, 12(5), 7-7.

[31] Savage, R. A., Lipp, O. V., Craig, B. M., Becker, S. I., \&Horstmann, G. (2013). In search of the emotional face: Anger versus happiness superiority in visual search. Emotion, 13(4), 758.

[32] Rhodes, G. (2006). The evolutionary psychology of facial beauty. Annu. Rev. Psychol., 57, 199-226.

[33] Craig, B. M., Becker, S. I., \&Lipp, O. V. (2014). Different faces in the crowd: A happiness superiority effect for schematic faces in heterogeneous backgrounds. Emotion, 14(4), 794.

[34] Ambady, N., Weisbuch, M., Calder, A., Rhodes, G., Johnson, M., \&Haxby, J. (2011). On perceiving facial expressions: The role of culture and context. Oxford handbook of face perception, 479-488.

[35] Fan, Q., Sui, X., \& Fu, Y. (2014). An Eye-tracking Research on Featural, Configural and Holistic Processing Strategies in Face Perception. Acta PsychologicaSinica, 46(8), 1062-1071. 